\title{
The monocyte to red blood cell count ratio is a strong predictor of postoperative survival in colorectal cancer patients: The Fujian prospective investigation of cancer (FIESTA) study
}

\author{
Feng Peng ${ }^{*}$, Dan $\mathrm{Hu}^{2 *}$, Xiandong Lin², Gang Chen², Binying Liang 3 , Chao $\mathrm{Li}^{2}$, Yan Chen ${ }^{4}$, Zhaolei Cui ${ }^{4}$, \\ Hejun Zhang ${ }^{2}$, Jixiu Lin ${ }^{\natural}$, Xiongwei Zheng ${ }^{2}$, Wenquan Niu ${ }^{5}$ \\ 1. Department of Cardiology, The First Affiliated Hospital of Fujian Medical University, Fuzhou, Fujian, China; \\ 2. Department of Pathology, Fujian Provincial Cancer Hospital, The Affiliated Hospital of Fujian Medical University, Fuzhou, Fujian, China; \\ 3. Department of Medical Record, Fujian Provincial Cancer Hospital, The Affiliated Hospital of Fujian Medical University, Fuzhou, Fujian, China; \\ 4. Department of Clinical Laboratory, Fujian Provincial Cancer Hospital, The Affiliated Hospital of Fujian Medical University, Fuzhou, Fujian, China; \\ 5. State Key Laboratory of Medical Genomics, Rui Jin Hospital, Shanghai Jiao Tong University School of Medicine, Shanghai, China. \\ *Shared first authors.
}

$\triangle$ Corresponding authors: Wenquan Niu, Ph.D., Rui Jin Second Road 197, New Huang Pu District, Shanghai 200025, China. Phone: +86-21-6437 0045 ext. 610905. Fax: +86-21-6433 3548. E-mail: niuwenquan_shcn@163.com or nwq11588@rjh.com.cn Xiongwei Zheng, M.D. Ph.D., Fu Ma Road 420, Jin An District, Fuzhou 350014, Fujian, China. Phone: +86-591-8364 3149. Fax: +86-591-8392 8767. E-mail: agu1960@126.com Jinxiu Lin, M.D. Ph.D., Chazhong Road 20, Tai Jiang District, Fuzhou 350005, Fujian, China. Phone: +86-591-8798 1637. Fax: +86-591-8798 1635. E-mail: linjinxiu@medmail.com.cn.

(c) Ivyspring International Publisher. This is an open access article distributed under the terms of the Creative Commons Attribution (CC BY-NC) license (https://creativecommons.org/licenses/by-nc/4.0/). See http://ivyspring.com/terms for full terms and conditions.

Received: 2016.10.19; Accepted: 2016.12.23; Published: 2017.03.12

\begin{abstract}
Background and Aims: We sought to evaluate the prognosis of preoperative blood routine parameters for the mortality of colorectal cancer patients after surgery by eliciting a subset of data from the ongoing Fujian prospective investigation of cancer (FIESTA) study.

Methods: 1318 colorectal cancer patients with completed survival data were enrolled between 2000 and 2008. Effect-size estimates are expressed as hazard ratio (HR) and $95 \%$ confidence interval $(\mathrm{Cl})$.

Results: The median follow-up time was 58.6 months. Elevated levels of neutrophil (adjusted $\mathrm{HR}, 95 \% \mathrm{Cl}, \mathrm{P}$ : $1.22,1.06-1.41,0.006)$ and monocyte $(1.32,1.06-1.65,0.013)$ were significantly associated with an increased risk of colorectal cancer mortality, whereas that of lymphocyte $(0.60,0.44-0.82,0.001)$ and red blood cell count $(0.20,0.09-0.43,<0.001)$ were significantly associated with a reduced risk. Additionally, remarkable significance was reached for the neutrophil-to-lymphocyte ratio $(1.12,1.06-1.19,<0.001)$ and lymphocyte-to-monocyte ratio $(0.60,0.46-0.79,<0.001)$. Based on individual effect-estimates, a new derivate, monocyte to red blood cell count ratio namely MRR was created, and its association with colorectal cancer mortality was strikingly significant $(1.48,1.18-1.85,0.001)$. Notably, elevated MRR was significantly associated with the mortality of early stage colorectal cancer, especially in patients with stage I-II $(1.63,1.04-2.56,0.034)$, invasion depth T1-T2 $(2.85,1.45-5.61,0.002)$, regional lymph node metastasis N0 $(1.89,1.29-2.77,0.001)$ and tumor size $\leq 4.5 \mathrm{~cm}(1.84,1.25-2.70,0.002)$.

Conclusions: We created a new derivate MRR, which was superior over classic blood routine derivates, and importantly the MRR exhibited a stronger ability in predicting poor prognosis of colorectal cancer, especially at the early stage.
\end{abstract}

Key words: Colorectal cancer; Blood routine parameter; Prognosis; Survival; FIESTA study.

\section{Introduction}

Colorectal cancer is projected to be the fifth leading cause of cancer mortality in China [1]. The overall 5-year survival rate hinging upon staging differs substantially, ranging from $93.2 \%$ for stage I to $8.1 \%$ for stage IV [2]. Early detection of patients with colorectal cancer is therefore vital to improve its 
daunting mortality. During the last decade, considerable efforts have been devoted to identifying potential risk factors responsible for the onset and progression of colorectal cancer [3-5]. Many lifestyle factors have been established to increase the chance of developing colorectal cancer, including physical inactivity, low fruit and vegetable intake, overweight or obesity, alcohol consumption and tobacco use [6-8]. So far, a growing number of studies have demonstrated that certain blood routine parameters or derivates can better predict the postoperative survival in colorectal cancer patients. For example, the neutrophil-to-lymphocyte ratio (NLR) and platelet-to-lymphocyte ratio (PLR) were identified as two significant independent prognostic factors for colorectal cancer mortality [9, 10]. Further, a more recent meta-analysis of 15 studies and 3991 colorectal cancer patients suggested that elevated peripheral blood PLR was associated with the significant risk of cancer-related mortality, as well as poor tumor differentiation, increased infiltration depth and high relapse rate [11]. In fact, blood routine tests are simple and inexpensive, and thus screening for blood routine parameters will be practical in many clinical settings. However, one of the pressing problems facing clinicians now is that the significant findings between blood routine parameters and the prognosis of colorectal cancer are not consistently reproducible across studies [12, 13]. Many times, such irreproducibility may often attribute to inadequate statistical power and short follow-up periods. To fill this gap in knowledge, we sought to evaluate the prognostic utility of preoperative blood routine parameters for the risk of mortality among 1318 colorectal cancer patients who underwent radical resection by eliciting a subset of data from the ongoing Fujian prospective investigation of cancer (FIESTA) study [14-17].

\section{Methods}

\section{Study Patients}

All study patients were obtained from the ongoing FIESTA study initiated in January 2000. The FIESTA study is an ongoing prospective cohort study for common digestive system tumors, including esophageal cancer, gastric cancer and colorectal cancer [14-17], and the chief objective of the FIESTA study is to look for preoperative risk factors for cancer-specific mortality, which will help define rational targets to deter tumor progression and prolong the survival of cancer patients after surgery.

For the present study, the number of this cohort has increased annually by consecutively enrolling patients who were operable for colorectal cancer from the Department of Thoracic Surgery, Fujian Provincial Cancer Hospital. This study was officiated and approved by the research review boards of Fujian Provincial Cancer Hospital. All study patients were duly informed and signed written informed consent prior to enrollment.

\section{Inclusion Criteria}

By December 31, 2008, patients with colorectal cancer which was confirmed by preoperative biopsy or postoperative pathologic tests were included in the present study, and they must be Chinese of Han descent and without consanguinity. In addition, relapsed patients with colorectal cancer were excluded, as well as for patients receiving preoperative/postoperative chemotherapy or radiotherapy. A minimum follow-up period of 1 month was required.

\section{Tissue Collection}

Tissues at both cancer and nearby normal sites were resected from each patient, and they were formalin-fixed and paraffin-embedded for pathological analysis at the Department of Pathology, Fujian Provincial Cancer Hospital.

\section{Follow-up Survey}

Each year, all study patients were requested to pay following visits to the Out-Patient Department, Fujian Provincial Cancer Hospital within a predetermined time frame. If the patients failed to show in due time, we were trying to get ahold of them to record necessary information. Overall survival time was defined as the time from date of radical resection for colorectal cancer to date of death or date of latest follow-up in 2015, whichever came first.

\section{Blood Routine Test}

After admission, each patient was request to provide a fasting venous blood sample to measure blood routine parameters, including neutrophil, lymphocyte, monocyte, eosinophil, white blood cell count, red blood cell count, hemoglobin, red cell distribution width and platelet count by the SYSMEX XE-2100 Automatic Blood Cell Analyzer (Sysmex, Kobe, Japan) at the Clinical Laboratory, Fujian Provincial Cancer Hospital. NLR, PLR and lymphocyte-to-monocyte ratio (LMR) were derived from single parameters. The time period between the surgery for colorectal cancer and the abstraction of blood samples was within one week.

\section{Demographic and Clinical Characteristics}

Personal interviews coupled with structured questionnaires were used to gather demographic data, including date of birth, first onset age for 
colorectal cancer, sex, weight, height, smoking, drinking and family cancer history. Body mass index (BMI) was calculated as weight in kilograms divided by height in meters-squared. For smoking, the number of patients who never smoked and ever (formerly and currently) smoked was recorded. Likewise for drinking, the number of patients who never drank and ever (formerly and currently) drank was recorded.

Medical records were reviewed to collect clinicopathologic data including tumor-node-metastasis (TNM) stage (I, II, III and IV according to the $7^{\text {th }}$ Edition of the UICC/AJCC TNM Staging System [18]), tumor size (in centimeters), depth of invasion (T1, T2, T3 and T4), regional lymph node metastasis (N0, N1, N2 and N3), distant metastasis (M0 and M1), tumor differentiation, embolus and tumor size (in centimeters) were obtained from medical charts and pathological reports.

\section{Statistical Analysis}

Data were either expressed as median (interquartile range) for continuous variables or percent for categorical variables, and they were compared between the two groups by the Mann-Whitney test or $\mathrm{Chi}^{2}$ test where appropriate. The prognostic utility of preoperative blood routine parameters or derivates for colorectal cancer mortality was evaluated by hazard ratio (HR) and its 95\% confidence interval $(95 \% \mathrm{CI})$ on the basis of the Weibull proportional hazards regression model. Survival tree analysis was used to distinguish patients with divergent median survival time (MST) by the recursive-partitioning method as implemented in STREE software (http://c2s2.yale.edu/software/ stree/) developed by Zhang et al [19]. In concrete terms, survival tree analysis is implemented on the basis of a recursive partitioning algorithm. A general survival tree is developed layer by layer from a root node. The nodes within the same layer form a partition of the root node. The same splitting factor can be used by an offspring node as its ancestors. The process of recursive partitioning continues until the tree is saturated, which means that offspring nodes subject to further division cannot be split. Kaplan-Meier curves and log-rank tests were used to compare cumulative survival of colorectal cancer in carriers of different nodes in survival tree structure. Other statistical analyses were done by the STATA software (StataCorp, TX, USA, version 13.0 for the Windows).

\section{Results}

\section{Follow-up Evaluation}

There were 1458 colorectal cancer patients who received radical resection at Fujian Provincial Cancer Hospital and qualified for inclusion. As of late 2015, 104 patients were lost to follow-up, 27 patients died for reasons other than colorectal cancer, and 8 patients were followed up less than 1 month. So, 1318 colorectal cancer patients who had complete survival data were finally analyzed. The follow-up duration ranged from 1.4 months to 188.9 months (median 58.6 months), and the follow-up rate was $90.4 \%$. At the end of follow-up, 412 deaths (31.3\%) occurred.

\section{Baseline Characteristics}

The baseline characteristics of study patients with colorectal cancer before the operation are shown in Table 1. There were no significant differences between non-survivors and survivors in terms of age, sex, smoking, drinking and family cancer history (all $\mathrm{P}>0.10)$. Non-survivors had slightly higher BMI than survivors $(\mathrm{P}=0.028)$, as well as for neutrophil $(\mathrm{P}=0.018)$, monocyte $(\mathrm{P}=0.031)$ and red cell distribution width $(\mathrm{P}=0.016)$. By contrast, survivors had remarkably higher median levels of lymphocyte, red blood cell count, hemoglobin and LMR $(\mathrm{P}<0.01)$. No significance was seen for eosinophil, white blood cell count, platelet count and PLR $(P>0.10)$. The frequencies of invasion depth, regional lymph node metastasis, distant metastasis, TNM stage and tumor differentiation differed significantly between non-survivors and survivors $(\mathrm{P}<0.001)$. The number of lymph node metastasis was significantly higher in non-survivors than in survivors $(\mathrm{P}<0.001)$, and tumor size was comparable $(\mathrm{P}=0.146)$.

\section{Overall Risk Prediction}

The effect-size estimates of blood routine parameters or derivates for colorectal cancer mortality are summarized in Table 2. Estimates were calculated by per median increment of all parameters and with or without adjusting for age, sex, BMI, smoking, drinking and family cancer history under the Weibull proportional hazards regression model. Elevated levels of neutrophil (HR, 95\% CI, P: 1.22, 1.06-1.41, $0.006)$ and monocyte (1.32, 1.06-1.65, 0.013) were significantly associated with an increased risk of colorectal cancer mortality, whereas that of lymphocyte $(0.60,0.44-0.82,0.001)$, red blood cell count $(0.20,0.09-0.43,<0.001)$ and hemoglobin $(0.51$, $0.30-0.85,0.011)$ were significantly associated with a reduced risk after adjustment. As expected, significance was reached for the three classic derivates (NLR, PLR and LMR), especially for the NLR (HR, 
95\% CI, P: 1.12, 1.06-1.19, <0.001) and LMR (0.60, $0.46-0.79,<0.001)$. Furthermore, in view of the magnitude of effect-size estimates, a new derivate, monocyte to red blood cell count ratio namely the MRR was created, and its association with colorectal cancer mortality was strikingly significant (HR, 95\% CI, P: 1.49, 1.20-1.86, <0.001 before adjustment; 1.48, $1.18-1.85,0.001$ after adjustment).

Table 1. The baseline characteristics of cohort patients

\begin{tabular}{|c|c|c|c|}
\hline Characteristics & $\begin{array}{l}\text { Non-survivors } \\
(\mathrm{n}=412)\end{array}$ & Survivors $(\mathrm{n}=906)$ & $\mathrm{P}$ \\
\hline Age (years) & $56(45,67)$ & $57(48,66)$ & 0.409 \\
\hline Males & $58.74 \%$ & $58.06 \%$ & 0.816 \\
\hline Body mass index $\left(\mathrm{kg} / \mathrm{m}^{2}\right)$ & $\begin{array}{l}23.13(21.20 \\
25.35)\end{array}$ & $22.89(20.81,24.83)$ & 0.028 \\
\hline Smoking & $10.35 \%$ & $11.15 \%$ & 0.675 \\
\hline Drinking & $2.78 \%$ & $2.61 \%$ & 0.864 \\
\hline Family cancer history & $9.09 \%$ & $6.65 \%$ & 0.127 \\
\hline Neutrophil (109/L) & $3.9(3.0,5.1)$ & $3.7(2.9,4.8)$ & 0.018 \\
\hline Lymphocyte $\left(10^{9} / \mathrm{L}\right)$ & $1.7(1.3,2.1)$ & $1.9(1.5,2.2)$ & $<0.001$ \\
\hline Monocyte (109/L) & $0.5(0.4,0.7)$ & $0.5(0.4,0.6)$ & 0.031 \\
\hline Eosinophil $\left(10^{9} / \mathrm{L}\right)$ & $0.1(0.1,0.3)$ & $0.2(0.1,0.3)$ & 0.254 \\
\hline $\begin{array}{l}\text { White blood cell count } \\
\left(10^{9} / \mathrm{L}\right)\end{array}$ & $6.4(5.4,7.9)$ & $6.4(5.3,7.7)$ & 0.427 \\
\hline Red blood cell count (1012/L) & $4.12(3.77,4.53)$ & $4.19(3.87,4.60)$ & 0.006 \\
\hline Hemoglobin $(\mathrm{g} / \mathrm{L})$ & $124(109,136)$ & $126(113,138)$ & 0.009 \\
\hline $\begin{array}{l}\text { Red cell distribution width } \\
(\%)\end{array}$ & $13.4(12.5,14.8)$ & $13.1(12.4,14.3)$ & 0.016 \\
\hline Platelet count $\left(10^{9} / \mathrm{L}\right)$ & $245(197,313)$ & $255(205,313)$ & 0.198 \\
\hline $\begin{array}{l}\text { Neutrophil-to-lymphocyte } \\
\text { ratio }\end{array}$ & $2.26(1.62,3.42)$ & $2.00(1.53,2.64)$ & $<0.001$ \\
\hline Platelet-to-lymphocyte ratio & $\begin{array}{l}148.18(105.65 \\
200.15)\end{array}$ & $\begin{array}{l}138.89(105.00, \\
182.73)\end{array}$ & 0.105 \\
\hline $\begin{array}{l}\text { Lymphocyte-to-monocyte } \\
\text { ratio }\end{array}$ & $3.25(2.39,4.55)$ & $3.80(2.80,4.75)$ & $<0.001$ \\
\hline Invasion depth & & & $<0.001$ \\
\hline $\mathrm{T} 1$ & $2.75 \%$ & $6.04 \%$ & \\
\hline $\mathrm{T} 2$ & $6.25 \%$ & $16.92 \%$ & \\
\hline T3 & $41.75 \%$ & $45.68 \%$ & \\
\hline $\mathrm{T} 4$ & $49.25 \%$ & $31.36 \%$ & \\
\hline $\begin{array}{l}\text { Regional lymph node } \\
\text { metastasis }\end{array}$ & & & $<0.001$ \\
\hline No & $33.50 \%$ & $61.18 \%$ & \\
\hline N1 & $27.50 \%$ & $22.60 \%$ & \\
\hline N2 & $39.00 \%$ & $16.21 \%$ & \\
\hline Distant metastasis & $21.36 \%$ & $0.24 \%$ & $<0.001$ \\
\hline TNM stage & & & $<0.001$ \\
\hline I & $5.78 \%$ & $18.84 \%$ & \\
\hline II & $21.61 \%$ & $42.18 \%$ & \\
\hline III & $51.51 \%$ & $38.86 \%$ & \\
\hline IV & $21.11 \%$ & $0.21 \%$ & \\
\hline Differentiation & & & $<0.001$ \\
\hline High & $2.75 \%$ & $4.85 \%$ & \\
\hline Moderate & $66.25 \%$ & $76.69 \%$ & \\
\hline Low & $31.00 \%$ & $18.46 \%$ & \\
\hline Embolus & $38.25 \%$ & $17.76 \%$ & $<0.001$ \\
\hline Tumor size $(\mathrm{cm})$ & $5.0(3.5,6.0)$ & $4.5(3.5,6.0)$ & 0.146 \\
\hline $\begin{array}{l}\text { Number of lymph node } \\
\text { metastasis }\end{array}$ & $2(0,6)$ & $0(0,2)$ & $<0.001$ \\
\hline
\end{tabular}

Data is expressed as median (inter-quartile range) or percent where appropriate. *P was calculated by the Mann-Whitney $U$ test for continuous variables and the $\mathrm{Chi}^{2}$ test for categorical variables.

\section{Survival Tree Analysis}

Figure 1 provides the structure of survival tree analysis on blood routine parameters or derivates, demographic and clinical characteristics. The first-tier factor in the survival tree was TNM stage, with patients with stage I-III having remarkably longer MST than patients with stage IV (61.2 months vs. 15.6 months, Log-rank test: $\mathrm{P}<0.001)$. The second-tier factor in patients with stage I-III was regional lymph node metastasis, followed by the NLR and LNM, further by MRR, LMR, red cell distribution width, platelet count and the NLR. Notably, patients in node 3 had longer MST than its twin node (63.8 months vs. 42.7 months, Log-rank test: $\mathrm{P}<0.001)$. The MST between node 4 and node 5 , node 6 and node 7 , node 8 and node 9 differed significantly (Log-rank test: $\mathrm{P}<0.001)$. Further Kaplan-Meier curves were displayed to examine the differences in cumulative survival for the nodes incorporating 50 or more patients, which validated the discrimination ability of survival tree analysis (Figure 1).

\section{Risk Prediction by Clinicopathologic Features}

Shown in Table 3 are the effect-size estimates of four blood routine derivates (NLR, PLR, LMR and MRR) for colorectal cancer mortality by clinicopathologic features after adjusting for confounding factors mentioned above. The prediction of the NLR was strongly reinforced in patients with stage III-IV (HR, 95\% CI, P: 1.21, 1.12-1.30, <0.001), invasion depth T3-T4 $(1.12,1.05-1.19,<0.001)$ and negative embolus $(1.32,1.17-1.49,<0.001)$. The prediction estimates of the PLR were comparable between the same clinicopathologic subgroups, except in patients with tumor size $>4.5 \mathrm{~cm}(\mathrm{HR}, 95 \%$ CI, P: 1.26, 1.05-1.51, 0.014). For the LMR, reinforced prediction was identified in patients with invasion depth T1-T2 (HR, 95\% CI, P: 0.35, 0.13-0.96, 0.042), high or moderate differentiation $(0.57,0.41-0.80,0.001)$ and negative embolus $(0.45,0.28-0.71,0.001)$. Elevated MRR was significantly associated with the early stage of colorectal cancer, especially in patients with stage I-II (HR, 95\% CI, P: 1.63, 1.04-2.56, 0.034), invasion depth T1-T2 (2.85, 1.45-5.61, 0.002), regional lymph node metastasis N0 $(1.89,1.29-2.77,0.001)$ and tumor size $\leq 4.5 \mathrm{~cm}(1.84,1.25-2.70,0.002)$. In addition, the MRR can better predict the survival of patients with high or moderate differentiation $(1.53,1.16-2.02,0.003)$ and positive embolus $(2.04,1.28-3.23,0.003)$. 

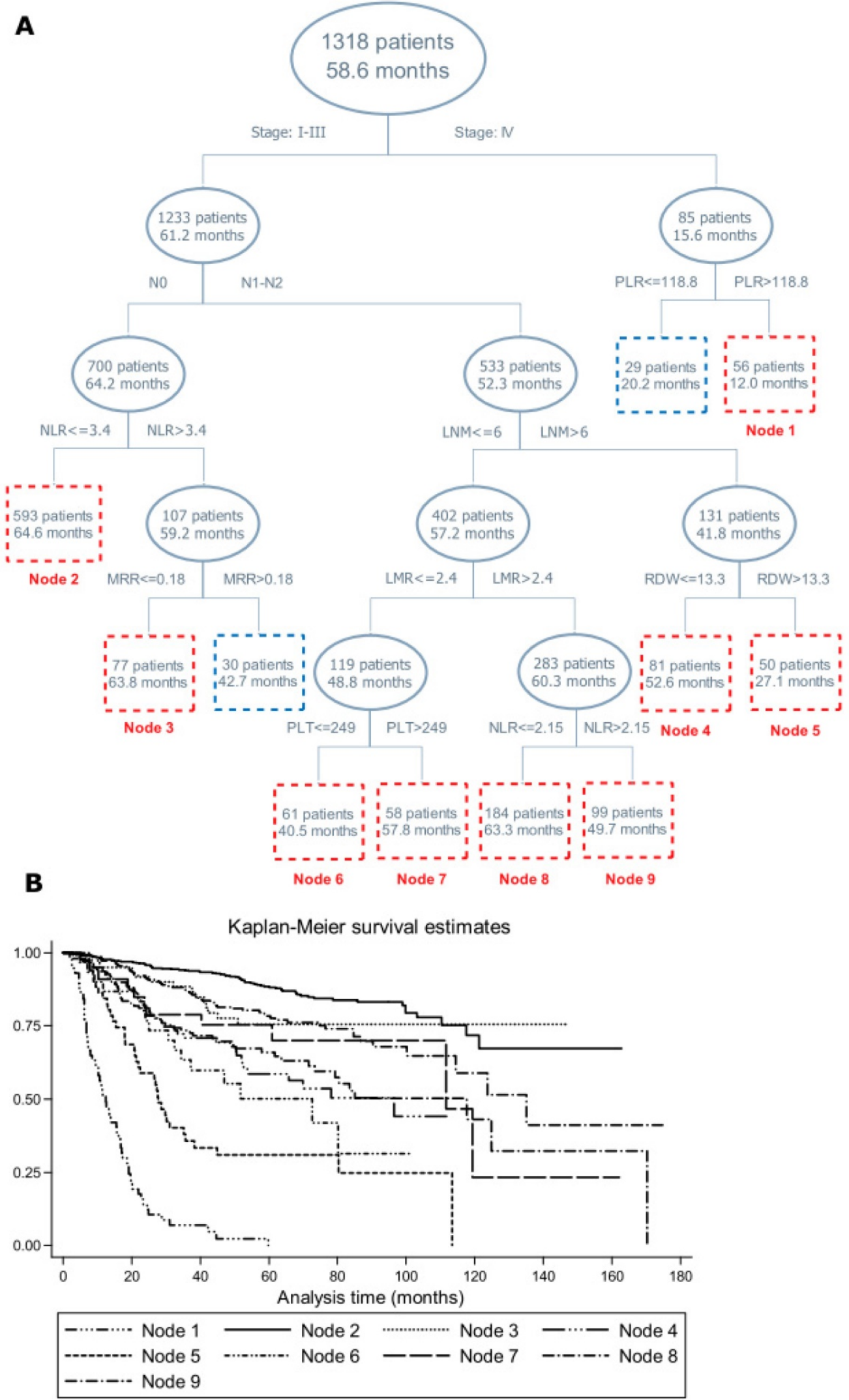

Figure 1. Survival tree structure (A) and Kaplan-Meier curve (B) of blood routine parameters or derivates, demographic and clinical characteristics on the prognosis of colorectal cancer mortality. Abbreviations: NLR, Neutrophil-to-lymphocyte ratio; PLR, Platelet-to-lymphocyte ratio; LMR, lymphocyte-to-monocyte ratio; MRR, monocyte-to-red blood cell count ratio; LNM, lymph node metastasis; PLT, platelet count; RDW, red cell distribution width. The upper number in the box refers to the number of patients, and the lower number refers to the median survival time. 
Table 2. Effect-size estimates of blood routine parameters for colorectal cancer mortality

\begin{tabular}{llll}
\hline Blood routine parameters & Median & HR, 95\% CI, P & HR, 95\% CI, P $^{*}$ \\
\hline Single parameters & & & $1.22,1.06-1.41,0.006$ \\
Neutrophil $\left(10^{9} / \mathrm{L}\right)$ & 3.7 & $1.21,1.06-1.40,0.007$ & $0.60,0.44-0.82,0.001$ \\
Lymphocyte $\left(10^{9} / \mathrm{L}\right)$ & 1.8 & $0.59,0.44-0.80,0.001$ & $1.32,1.06-1.65,0.013$ \\
Monocyte $\left(10^{9} / \mathrm{L}\right)$ & 0.5 & $1.33,1.08-1.65,0.008$ & $1.02,0.91-1.15,0.705$ \\
Eosinophil $\left(10^{9} / \mathrm{L}\right)$ & 0.2 & $1.02,0.91-1.15,0.729$ & $1.11,0.90-1.36,0.349$ \\
White blood cell count $\left(10^{9} / \mathrm{L}\right)$ & 6.4 & $1.11,0.91-1.36,0.301$ & $0.20,0.09-0.43,<0.001$ \\
Red blood cell count $\left(10^{12} / \mathrm{L}\right)$ & 4.17 & $0.25,0.12-0.52,<0.001$ & $0.51,0.30-0.85,0.011$ \\
Hemoglobin $(\mathrm{g} / \mathrm{L})$ & 125 & $0.54,0.33-0.88,0.014$ & $1.00,0.89-1.13,0.990$ \\
Red cell distribution width $(\%)$ & 13.2 & $1.00,0.90-1.13,0.944$ & $0.93,0.71-1.21,0.583$ \\
Platelet count $\left(10^{9} / \mathrm{L}\right)$ & 252 & $0.92,0.71-1.20,0.554$ & $1.12,1.06-1.19,<0.001$ \\
Derivates & & & $1.16,1.02-1.32,0.028$ \\
Neutrophil-to-lymphocyte ratio & 2.05 & $1.12,1.06-1.19,<0.001$ & $0.60,0.46-0.79,<0.001$ \\
Platelet-to-lymphocyte ratio & 140 & $1.18,1.03-1.34,0.013$ & $1.48,1.18-1.85,0.001$ \\
Lymphocyte-to-monocyte ratio & 3.62 & $0.59,0.45-0.77,<0.001$ & $1.49,1.20-1.86,<0.001$ \\
Monocyte-to-red blood cell count ratio & 0.13 & & \\
\hline
\end{tabular}

Abbreviations: HR, hazard ratio; 95\% CI, 95\% confidence interval. *P was adjusted for age, sex, body mass index, smoking, drinking and family cancer history.

Table 3. Effect-size estimates of four blood routine derivates for colorectal cancer mortality by clinicopathologic features

\begin{tabular}{|c|c|c|c|c|c|}
\hline \multirow[t]{2}{*}{ Subgroups } & & \multicolumn{4}{|l|}{ HR, 95\% CI, $\mathrm{P}^{*}$} \\
\hline & & NLR (per 2.05+) & PLR (per 140+) & LMR (per 3.62+) & MRR (per 0.13+) \\
\hline \multirow[t]{2}{*}{ TNM stage } & I-II & $1.10,0.99-1.21,0.064$ & $1.23,1.01-1.51,0.045$ & $0.58,0.35-0.98,0.042$ & $1.63,1.04-2.56,0.034$ \\
\hline & III-IV & $1.21,1.12-1.30,<0.001$ & $1.25,1.03-1.52,0.024$ & $0.64,0.46-0.88,0.005$ & $1.38,1.07-1.78,0.014$ \\
\hline \multirow[t]{2}{*}{ Invasion depth } & TI-T2 & $1.06,0.82-1.36,0.676$ & $1.04,0.68-1.60,0.848$ & $0.35,0.13-0.96,0.042$ & $2.85,1.45-5.61,0.002$ \\
\hline & T3-T4 & $1.12,1.05-1.19,<0.001$ & $1.15,0.99-1.34,0.065$ & $0.67,0.51-0.87,0.003$ & $1.31,1.03-1.67,0.029$ \\
\hline \multirow[t]{2}{*}{ Regional LNM } & No & $1.13,1.02-1.24,0.015$ & $1.23,1.01-1.49,0.037$ & $0.52,0.32-0.84,0.008$ & $1.89,1.29-2.77,0.001$ \\
\hline & N1-N2 & $1.14,1.06-1.23,<0.001$ & $1.23,1.00-1.51,0.047$ & $0.66,0.48-0.92,0.013$ & $1.31,1.00-1.72,0.053$ \\
\hline \multirow[t]{2}{*}{ Distant metastasis } & Negative & $1.11,1.04-1.19,0.003$ & $1.12,0.96-1.31,0.145$ & $0.67,0.50-0.90,0.007$ & $1.39,1.07-1.81,0.015$ \\
\hline & Positive & $1.14,0.98-1.33,0.090$ & $1.21,0.90-1.63,0.209$ & $0.72,0.37-1.39,0.331$ & $1.31,0.73-2.33,0.361$ \\
\hline \multirow[t]{2}{*}{ Differentiation } & High/moderate & $1.11,1.01-1.21,0.025$ & $1.16,1.00-1.36,0.057$ & $0.57,0.41-0.80,0.001$ & $1.53,1.16-2.02,0.003$ \\
\hline & Low & $1.10,1.02-1.20,0.017$ & $1.16,0.87-1.55,0.303$ & $0.76,0.54-1.07,0.113$ & $1.29,0.85-1.95,0.230$ \\
\hline \multirow[t]{2}{*}{ Embolus } & Negative & $1.32,1.17-1.49,<0.001$ & $1.38,1.12-1.70,0.002$ & $0.45,0.28-0.71,0.001$ & $1.54,1.09-2.18,0.015$ \\
\hline & Positive & $1.10,0.98-1.23,0.114$ & $1.39,1.00-1.94,0.052$ & $0.75,0.50-1.13,0.168$ & $2.04,1.28-3.23,0.003$ \\
\hline \multirow[t]{2}{*}{ Tumor size } & $\leq 4.5 \mathrm{~cm}$ & $1.10,0.99-1.22,0.082$ & $1.01,0.80-1.27,0.947$ & $0.61,0.40-0.91,0.015$ & $1.84,1.25-2.70,0.002$ \\
\hline & $>4.5 \mathrm{~cm}$ & $1.11,1.03-1.20,0.004$ & $1.26,1.05-1.51,0.014$ & $0.67,0.48-0.95,0.023$ & $1.25,0.92-1.68,0.150$ \\
\hline
\end{tabular}

Abbreviations: HR, hazard ratio; 95\% CI, 95\% confidence interval; NLR, Neutrophil-to-lymphocyte ratio; PLR, Platelet-to-lymphocyte ratio; LMR, lymphocyte-to-monocyte ratio; MRR, monocyte-to-red blood cell count ratio; TNM, tumor-node-metastasis; LNM, lymph node metastasis. *P was adjusted for age, sex, body mass index, smoking, drinking and family cancer history.

\section{Discussion}

The major finding of the present study was that we consolidated the prognostic utility of three classic blood routine derivates, especially NLR and LMR, for patients with postoperative colorectal cancer during a median follow-up of 58.6 months. The second major finding was that we created a new derivate MRR based on monocyte and red blood cell count, and this derivate exhibited a stronger ability in predicting the poor prognosis of colorectal cancer, especially at the early postoperative period. To the authors' knowledge, this is so far the largest and longest prospective cohort study that has evaluated the prognosis of preoperative blood routine parameters for colorectal cancer mortality.

A large number of cohort studies have shown that blood routine parameters, especially three classic derivates NLR, PLR and LMR, are strong predictors of postoperative survival in colorectal cancer patients [20-24]. However, some but not all studies reported a positive association between these derivates and the prognosis of colorectal cancer. For example, the majority of studies found that elevated PLR was a significant predictor of poor survival in colorectal cancer patients [25, 26], whereas other groups reported the neutral impact of PLR on colorectal cancer survival [27]. In general, small sample sizes and short follow-up periods may contribute to these diverging findings. To make a convincing estimate, we resorted to a subset of the data from the ongoing FIESTA study [14-17] and evaluated the prognostic utility of preoperative blood routine parameters for the risk of mortality among 1318 colorectal cancer patients who underwent radical resection during a median follow-up of 58.6 months. Such a larger sample size and a longer follow-up period enable us to have sufficient power to detect a long-term prognostic impact of blood routine parameters on colorectal cancer survival. It is noteworthy that we consolidated the prognostic utility of three classic blood routine derivates, especially NLR and LMR, for 
patients with postoperative colorectal cancer. This convincing finding may help clear up the confusion of scientific community and represent a further step towards improving the prognosis of colorectal cancer.

Besides, based on individual estimates of blood routine parameters, we created a new derivate, MRR, the ratio of monocyte to red blood cell count, and the predictive ability of this derivate is superior over that of three classic derivates under study. The physiological rationale underlying MRR is obvious. In fact, monocyte circulating in the blood is a precursor of tumor-associated macrophages, which are increasingly recognized as major players in the tumor microenvironment and cancer-related inflammation [28, 29]. Emerging data have suggested that high infiltration of tumor-associated macrophages is associated with poor prognosis of patients with digestive cancers [30, 31]. Tumor-associated macrophages can produce many growth factors such as epidermal growth factors and transforming growth factors for tumor cells and nascent blood vessels, and thus facilitate tumor proliferation [32]. In addition, red blood cells circulate in blood and carry oxygen throughout the body. A lack of red blood cells in blood is a major characteristic of anemia, a frequent complication in cancer patients. As indicated by a long-term retrospective study in 758 patients, reduced red blood cell count can predict poor survival in postoperative patients with liver cancer [33]. Another study suggested that low red blood cell levels of deglycating enzymes may be involved in the malignant transformation of colon mucosa [34]. On the basis of these observations, it is reasonable to believe that the ratio of monocyte to red blood cell count, namely MRR, is a promising prognostic factor for the poor survival of colorectal cancer patients. The MRR, coupled with current screening strategies can help early identify patients with postoperative colorectal cancer who will experience poor survival and finally improve their prognosis.

It is also worth noting that the predictive ability of NLR, LMR and MRR differed between postoperative patients with the early and late stages of colorectal cancer, as reflected in our stratified analyses (Table 3). The NLR is identified as an indicator of inflammation and immune function, as well as a prognostic factor for cancer at many sites, including colon and rectum [13, 35]. Our findings demonstrated that elevated preoperative NLR had a better predicting effect on the late-stage than the early-stage of colorectal cancer, which accorded with the findings in recent studies $[9,36]$. By contrast, LMR served as a favorable prognostic factor for postoperative colorectal cancer patients, and its favorable effect was more obvious on the early-stage of colorectal cancer than its late-stage. A latest meta-analysis by Song et al indicated that reduced preoperative LMR was associated with a worse survival in colorectal cancer patients, especially with reported metastases [37], which at least partly backed up the stratified findings of this study. As an extension, the only blood routine marker shared in common between LMR and the newly-created MRR was monocyte. The promising physiological role of monocyte in carcinogenesis has been discussed above. There is compelling clinical evidence that the number of preoperative blood monocytes can predict poor prognosis of various types of cancer. In the present study, the predictive ability of the MRR for colorectal cancer was more obvious in patients at the early-stage than the late-stage, in agreement with the LMR. Although the elucidation of the physiological mechanisms underlying the preoperative MRR and colorectal cancer survival is beyond the scope of this study, this agreement in prognostic stages raises a presumption that blood monocytes, if involved, play a critical role in the early carcinogenesis of colorectal cancer, possibly involving the microenvironmental regulation of tumor progression and cancer-related inflammation $[28,29,38]$.

Our study has several limitations. First, this was a single-center study, and so our findings need consistent validation in other cohorts before extrapolation. However, the single-center design may represent an important indicator of daily clinical practice. Second, all study patients with colorectal cancer were enrolled between the year 2000 and 2008, and during this 9-year period remarkable advances in surgical techniques might yield a possible bias, which may underestimate the prediction of blood routine parameters on colorectal cancer mortality. Third, routine blood parameters were measured only before the surgery for colorectal cancer, and to explore the changes of blood parameters is beyond the scope of our present study but certainly require further investigations. Fourth, the findings identified in this study were merely based on colorectal cancer patients who are suitable for radical resection, and therefore cannot be directly extrapolated to general populations.

In conclusion, we not only consolidated the prognostic utility of three classic blood routine derivates for patients with postoperative colorectal cancer, but also created a new derivate MRR based on monocyte and red blood cell count, showing that this derivate exhibited a stronger ability in predicting the poor prognosis of colorectal cancer, especially at the early postoperative period. Pending successful validation, our findings may usher future personalized medicine by measuring blood routine 
parameters preoperatively to identify colorectal cancer patients who might have a poor survival probability and treat them with optimal regimens.

\section{Acknowledgements}

This study was financially supported by the Natural Science Foundation of Fujian Province (grant nos. 2015J01451, 2016J01508, 2016J01513), the Training Project for Young and Middle-Aged Core Talents of Health System of Fujian Province (grant nos. 2015-ZQN-JC-7, 2015-ZQN-JC-22), the Science and Technology Plan Projects of Fujian Province (grant no. 2014Y0019), the National Clinical Key Specialty Construction Program of China (grant no. 2013-544) and the Ministry of Health P.R. China (grant no. WKJ2016-2-05).

\section{Contributors}

W.N., X.Z., F.P. drafted the protocol; D.H., X.L., Y.C., B.L., G.C., Z.C. obtained statutory and ethics approvals; D.H., X.L., Y.C., B.L., G.C., Z.C., C.L., H.Z., J.L. contributed to data acquisition; D.H., F.P., J.L., X.Z., W.N. had access to all raw data; D.H., X.L., W.N. did the data preparation, quality control and analyses, and checked the results; W.N., F.P., D.H. drafted the report. All authors contributed to writing the final report and approved the version to be published.

\section{Competing Interests}

The authors have declared that no competing interest exists.

\section{References}

1. Chen W, Zheng R, Baade PD, Zhang S, Zeng H, Bray F, et al. Cancer statistics in China, 2015. CA Cancer J Clin. 2016; 66: 115-32.

2. O'Connell JB, Maggard MA, Ko CY. Colon cancer survival rates with the new American Joint Committee on Cancer sixth edition staging. J Natl Cancer Inst. 2004; 96: 1420-5.

3. Wang ZH, Fang JY. Colorectal Cancer in Inflammatory Bowel Disease: Epidemiology, Pathogenesis and Surveillance. Gastrointest Tumors. 2014; 1: $146-54$.

4. Altobelli E, Angeletti PM, Latella G. Role of Urinary Biomarkers in the Diagnosis of Adenoma and Colorectal Cancer: A Systematic Review and Meta-Analysis. J Cancer. 2016; 7: 1984-2004.

5. Basnet S, Zhang ZY, Liao WQ, Li SH, Li PS, Ge HY. The Prognostic Value of Circulating Cell-Free DNA in Colorectal Cancer: A Meta-Analysis. J Cancer. 2016; 7: 1105-13.

6. Steffen A, MacInnis RJ, Joshy G, Giles GG, Banks E, Roder D. Development and validation of a risk score predicting risk of colorectal cancer. Cancer Epidemiol Biomarkers Prev. 2014; 23: 2543-52.

7. Yarnall JM, Crouch DJ, Lewis CM. Incorporating non-genetic risk factors and behavioural modifications into risk prediction models for colorectal cancer. Cancer Epidemiol. 2013; 37: 324-9.

8. Troy JD, Hartge P, Weissfeld JL, Oken MM, Colditz GA, Mechanic LE, et al. Associations between anthropometry, cigarette smoking, alcohol consumption, and non-Hodgkin lymphoma in the Prostate, Lung, Colorectal, and Ovarian Cancer Screening Trial. Am J Epidemiol. 2010; 171: 1270-81.

9. Zou ZY, Liu HL, Ning N, Li SY, Du XH, Li R. Clinical significance of pre-operative neutrophil lymphocyte ratio and platelet lymphocyte ratio as prognostic factors for patients with colorectal cancer. Oncol Lett. 2016; 11: 2241-8.

10. You J, Zhu GQ, Xie L, Liu WY, Shi L, Wang OC, et al. Preoperative platelet to lymphocyte ratio is a valuable prognostic biomarker in patients with colorectal cancer. Oncotarget. 2016.

11. Tan D, Fu Y, Su Q, Wang H. Prognostic role of platelet-lymphocyte ratio in colorectal cancer: A systematic review and meta-analysis. Medicine (Baltimore). 2016; 95: e3837.
12. Malietzis $G$, Giacometti $M$, Kennedy $R H$, Athanasiou $T$, Aziz $O$, Jenkins JT. The emerging role of neutrophil to lymphocyte ratio in determining colorectal cancer treatment outcomes: a systematic review and meta-analysis. Ann Surg Oncol. 2014; 21: 3938-46.

13. Li MX, Liu XM, Zhang XF, Zhang JF, Wang WL, Zhu Y, et al. Prognostic role of neutrophil-to-lymphocyte ratio in colorectal cancer: a systematic review and meta-analysis. Int J Cancer. 2014; 134: 2403-13.

14. Hu D, Lin X, Chen Y, Chang Q, Chen G, Li C, et al. Preoperative blood-routine markers and prognosis of esophageal squamous cell carcinoma: The Fujian prospective investigation of cancer (FIESTA) study. Oncotarget. 2016.

15. Hu D, Peng F, Lin X, Chen G, Liang B, Li C, et al. The elevated preoperative fasting blood glucose predicts a poor prognosis in patients with esophageal squamous cell carcinoma: The Fujian prospective investigation of cancer (FIESTA) study. Oncotarget. 2016; 7: 65247-56.

16. Hu D, Peng F, Lin X, Chen G, Zhang H, Liang B, et al. Preoperative Metabolic Syndrome Is Predictive of Significant Gastric Cancer Mortality after Gastrectomy: The Fujian Prospective Investigation of Cancer (FIESTA) Study. EBioMedicine. 2017; 15: 73-80.

17. Peng F, Hu D, Lin X, Chen G, Liang B, Zhang H, et al. Preoperative metabolic syndrome and prognosis after radical resection for colorectal cancer: The Fujian prospective investigation of cancer (FIESTA) study. Int J Cancer. 2016; 139: 2705-13.

18. Edge SB, Compton CC. The American Joint Committee on Cancer: the 7th edition of the AJCC cancer staging manual and the future of TNM. Ann Surg Oncol. 2010; 17: 1471-4

19. Zhang HP, Singer B. Recursive Partitioning and Applications. Springer, New York. 2010.

20. Kaneko M, Nozawa H, Sasaki K, Hongo K, Hiyoshi M, Tada N, et al. Elevated neutrophil to lymphocyte ratio predicts poor prognosis in advanced colorectal cancer patients receiving oxaliplatin-based chemotherapy. Oncology. 2012; 82: 261-8.

21. Maeda K, Shibutani M, Otani H, Nagahara H, Ikeya T, Iseki Y, et al. Inflammation-based factors and prognosis in patients with colorectal cancer. World J Gastrointest Oncol. 2015; 7: 111-7.

22. Chan JC, Chan DL, Diakos CI, Engel A, Pavlakis N, Gill A, et al. The Lymphocyte-to-Monocyte Ratio is a Superior Predictor of Overall Survival in Comparison to Established Biomarkers of Resectable Colorectal Cancer. Ann Surg. 2016.

23. Grenader T, Nash S, Adams R, Kaplan R, Fisher D, Maughan T, et al. Derived neutrophil lymphocyte ratio is predictive of survival from intermittent therapy in advanced colorectal cancer: a post hoc analysis of the MRC COIN study. Br J Cancer. 2016; 114: 612-5.

24. Azab B, Mohammad F, Shah N, Vonfrolio S, Lu W, Kedia S, et al. The value of the pretreatment neutrophil lymphocyte ratio vs. platelet lymphocyte ratio in predicting the long-term survival in colorectal cancer. Cancer Biomark. 2014; 14: 303-12.

25. Ozawa T, Ishihara S, Nishikawa T, Tanaka T, Tanaka J, Kiyomatsu T, et al. The preoperative platelet to lymphocyte ratio is a prognostic marker in patients with stage II colorectal cancer. Int J Colorectal Dis. 2015; 30: 1165-71.

26. Song A, Eo W, Lee S. Comparison of selected inflammation-based prognostic markers in relapsed or refractory metastatic colorectal cancer patients. World J Gastroenterol. 2015; 21: 12410-20.

27. He W, Yin C, Guo G, Jiang C, Wang F, Qiu H, et al. Initial neutrophil lymphocyte ratio is superior to platelet lymphocyte ratio as an adverse prognostic and predictive factor in metastatic colorectal cancer. Med Oncol. 2013; 30: 439.

28. Solinas G, Germano G, Mantovani A, Allavena P. Tumor-associated macrophages (TAM) as major players of the cancer-related inflammation. J Leukoc Biol. 2009; 86: 1065-73.

29. Chanmee $\mathrm{T}$, Ontong $\mathrm{P}$, Konno K, Itano N. Tumor-associated macrophages as major players in the tumor microenvironment. Cancers (Basel). 2014; 6: 1670-90.

30. Zhang J, Yan Y, Yang Y, Wang L, Li M, Wang J, et al. High Infiltration of Tumor-Associated Macrophages Influences Poor Prognosis in Human Gastric Cancer Patients, Associates With the Phenomenon of EMT. Medicine (Baltimore). 2016; 95: e2636.

31. Sugimura K, Miyata H, Tanaka K, Takahashi T, Kurokawa Y, Yamasaki M, et al. High infiltration of tumor-associated macrophages is associated with a poor response to chemotherapy and poor prognosis of patients undergoing neoadjuvant chemotherapy for esophageal cancer. J Surg Oncol. 2015; 111: $752-9$.

32. Erreni M, Mantovani A, Allavena P. Tumor-associated Macrophages (TAM) and Inflammation in Colorectal Cancer. Cancer Microenviron. 2011; 4 : 141-54.

33. Xie X, Yao M, Chen X, Lu W, Lv Q, Wang K, et al. Reduced red blood cell count predicts poor survival after surgery in patients with primary liver cancer. Medicine (Baltimore). 2015; 94: e577.

34. Notarnicola M, Caruso MG, Tutino V, Guerra V, Misciagna G. Low red blood cell levels of deglycating enzymes in colorectal cancer patients. World J Gastroenterol. 2011; 17: 329-33.

35. Jia J, Zheng X, Chen Y, Wang L, Lin L, Ye X, et al. Stage-dependent changes of preoperative neutrophil to lymphocyte ratio and platelet to lymphocyte ratio in colorectal cancer. Tumour Biol. 2015; 36: 9319-25.

36. Kubo H, Murayama Y, Arita T, Kuriu Y, Nakanishi M, Otsuji E. The Prognostic Value of Preoperative Neutrophil-to-Lymphocyte Ratio in Colorectal Cancer. World J Surg. 2016; 40: 2796-802. 
37. Song W, Wang K, Zhang RJ, Zou SB. Prognostic value of the lymphocyte monocyte ratio in patients with colorectal cancer: A meta-analysis. Medicine (Baltimore). 2016; 95: e5540.

38. Xiang J, Zhou L, Li X, Bao $\mathrm{W}$, Chen $\mathrm{T}, \mathrm{Xi}$ X, et al. Preoperative Monocyte-to-Lymphocyte Ratio in Peripheral Blood Predicts Stages, Metastasis, and Histological Grades in Patients with Ovarian Cancer. Transl Oncol. 2016; 10: 33-9. 